\title{
Resiliencia, género y rendimiento académico en jóvenes universitarios del Estado de Morelos
}

Resilience, gender and academic performance in young university students in the State of Morelos

\author{
Recepción del artículo: 08-11-2020 ～Aceptación del artículo: 16-01-2021
}

Dulce Areli, Gómez-Esquivel Universidad Autónoma del Estado de Morelos

(México).

dulce.gomez@uaem.edu.mx

https://orcid.org/0000-0002-6422-1983

Ulises, Delgado Sánchez

Universidad Autónoma del Estado de Morelos

(México).

ulises.delgado@uaem.mx

https://orcid.org/0000-0002-4318-0238

Fernanda Gabriela, Martínez Flores

Universidad Autónoma del Estado de Morelos

(México).

fer.martinez@uaem.mx

https://orcid.org/0000-0002-2974-0876

María Araceli, Ortiz-Rodríguez

Universidad Autónoma del Estado de Morelos

(México).

araceli.ortiz@uaem.mx

https://orcid.org/0000-0003-0847-0261

Rubén, Avilés Reyes

Universidad Autónoma del Estado de Morelos

(México).

ruben.avilesreyes@uaem.edu.mx

https://orcid.org/0000-0001-5328-3081

Para referenciar este artículo:

Gómez, D. A. Delgado, U., Martínez, F. G., Ortiz, M. A. y Avilés, R. (2021). Resiliencia, género y rendimiento académico en jóvenes universitarios del Estado de Morelos. Revista ConCiencia EPG, 6(1), 36-51. https://doi.org/10.32654/CONCIENCIAEP

\section{Resumen}

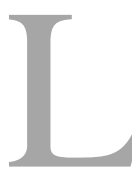

a resiliencia ha sido estudiada ampliamente en el campo de la psicología para definir aquellas personas que alcanzan el éxito pese a las circunstancias adversas. En este sentido, se realiza un estudio cuyo objetivo fue, evaluar la correlación entre resiliencia, rendimiento académico (RA), según el género en jóvenes universitarios, para lo cual se aplicó un diseño transversal analítico en donde participaron 51 estudiantes de la Licenciatura en Nutrición de la Universidad Autónoma del Estado de Morelos (UAEM) (18 hombres y 33 mujeres; de 21 a 32 años). Para evaluar las variables, se utilizó un cuestionario sociodemográfico, el inventario de anomia asiliente y resiliencia nomica (IAAR); y para determinar el RA se solicitó el promedio de calificaciones del semestre en curso. Los resultados evidencian que existe relación positiva entre resiliencia y RA, así como en los resultados de resiliencia y género, finalmente se evidencia que, los estudiantes del sexo masculino poseen niveles más altos de resiliencia que las mujeres y que el rendimiento académico se ve relacionado con la resiliencia.

Palabras Clave: Resiliencia; anomia asiliente; resiliencia nomica; rendimiento académico; género. 


\section{Summary}

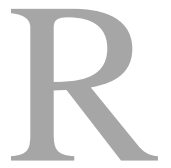

esilience has been widely studied in the field of psichology to define those who achieve success despite adeverse circumstances. Thus, a study is carried out whose objective was to evaluate the correlation between resilience and academic performance (RA), according to gender in university students, for which an analytical cross-sectional design was applied, with the participation of 51 undergraduate students in nutrition (18 men and 33 women; from 21 to 32 years old) from the Universidad Autónoma del Estado de Morelos (UAEM). To evaluate the variables, a sociodemographic questionare was used, the Asilient Anomie and Nomic Resilience Inventory (IAAR); and to determine the Academic Performance the grade point average for the current semester was requested. Results show that there is a positive relationship between resilience and Academic Performance, as well as in the results of resilience and gender, finally it is evidenced that male students have higher levels of resilience than women and that academic performance is related to the resilience.

Key Words: Resilience; asilient anomie; nomic resilience; Academic performance; Gender.

\section{Introducción}

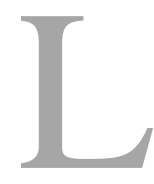

as personas resilientes se definen como aquellas que trabajan con fortaleza ante cualquier adversidad y utilizan sus propios recursos personales para reponerse y salir adelante, es decir, viven con constancia y capacidad para resistir y afrontar problemáticas que afectan su vida, su desempeño académico, vida familiar, personal, profesional y social (Goméz \& Rivas, 2017; Revilla, Martín \& Castro, 2016). Según Álvares y Caceres (2010) la resiliencia juega un papel importante en el contexto educativo, debido a que, el estudiante mide sus propias fortalezas frente a diversos retos y demandas, no sólo de tipo académico, sino también, a nivel biopsicosocial, para lo cual, saca a relucir sus habilidades, capacidades, etc., con lo que enfrenta las situaciones de manera eficaz.

Son estas capacidades las que se deben desarrollar en la educación, justamente, Gordon y Rouse (2001) refieren, que una de las maneras más apropiadas para acercarse al estudio de la resiliencia en la educación, es la resiliencia académica; en donde los estudiantes resilientes tienen una alta probabilidad de éxito a pesar de las adversidades contextuales (Martín \& Marsh, 2006), así mismo, describen que estos estudiantes son aquellos que, a pesar de tener eventos desafortunados en su vida, tienen una motivación elevada para alcanzar sus logros, el mismo que le permite elevar su desempeño en la escuela y permanecer en ella construyendo un ambiente propicio, y que el tránsito por la vida universitaria sea 
un proceso de aprendizaje, de motivación y determinación, para aumentar la cuota de resiliencia $y$ poder hacer frente a las exigencias y el ritmo de trabajo universitario.

Por otro lado, se cree que el género podría ser una variable que diferencia la construcción de la resiliencia, existen diversos estudios que indican que existe una asociación entre estas variables (González-Arratia et al., 2008; Morales \& Díaz, 2011). Estos autores señalan que hay diferencias en cuanto a los puntajes de resiliencia totales, e incluso se ha llegado a suponer que ser mujer es una variable "protectora" y que en su mayoría se ve asociada a la resiliencia.

También Grotberg (1995), señala que los niños varones son más inseguros y con dificultades de comunicación a comparación con las niñas quienes son más flexibles, lo cual propicia que en la resolución de conflictos posean una mayor habilidad interpersonal y fortalezas para afrontar la adversidad. Sin embargo, en un estudio realizado en niños y adolescentes con cáncer, observaron que el sexo masculino tenía mayores puntajes de resiliencia que las mujeres (Pintado \& Cruz, 2017).

Para tratar de dilucidar este tipo de cuestiones se realiza esta investigación con el objetivo analizar la correlación entre la resiliencia, el rendimiento académico y el género en jóvenes universitarios del Estado de Morelos.
Método

Participantes. En el estudio participaron 51 estudiantes (18 hombres y 33 mujeres; de 21 a 32 años) de la Licenciatura en Nutrición de la Universidad Autónoma del Estado de Morelos (UAEM). De los cuales 32 estudiantes cursaban séptimo semestre y 19 estaban en cuarto semestre de la licenciatura.

Instrumentos. La resiliencia se midió con el inventario de anomia asiliente y resiliencia nómica (IAAR) (Flores, 2013), La prueba consta de 35 ítems y tiene un coeficiente alfa de Cronbach de 0.86. El tiempo aproximado de duración de la prueba es de 10 a 15 minutos. Los niveles de resiliencia se evalúan en, muy alta (RMA) contiene una puntuación de 61-100; la resiliencia alta (RA) contiene un puntaje de 46-60; la resiliencia media (RM) de 31-45 puntos; la anomia baja (AB) de 0 -30 puntos; la anomia media (AM) de -1 a -30 puntos; la anomia alta (AA) de -31 a -45 puntos $\mathrm{y}$, por último, la anomia muy alta (AMA) -46 a 100 puntos.

Para las otras variables, se utilizó un registro de datos sociodemográficos que registran aspectos como el género, fecha de nacimiento, edad, nivel. socioeconómico y el semestre que están cursando. Y para evaluar el rendimiento académico (RA) se solicitó a la secretaría académica de la Facultad de Nutrición de la UAEM, el promedio de calificaciones del semestre en curso de los estudiantes, en donde 9 y 10 eran tomados como rendimiento alto y $7 \mathrm{y}$ 8 como rendimiento medio. 
Procedimiento. Antes del desarrollo de esta indagación se les concedió a los participantes una carta de consentimiento informado, la cual contenía los objetivos del estudio, así como el fin que tenía, por último, se les explicó que tenían la libertad de retirar su participación con tan solo avisar a los encargados de la investigación, si así lo consideraban. Una vez obtenidos los datos se analizaron en el programa Statistical Package for the Social Sciences (SPSS), versión 25. Para la descripción de los datos sociodemográficos se utilizaron frecuencias y porcentajes. Para el análisis de la resiliencia, RA y género se utilizaron las pruebas de $\mathrm{U}$ de Man Whitney, y la prueba de correlación lineal de Spearman (PCL), en la cual se considera una significancia estadística de los resultados de $\mathrm{p}<0.05$.

\section{Resultados}

Los datos sociodemográficos de nuestros participantes corresponden a un total de 51 estudiantes de la licenciatura en nutrición de la Universidad Autónoma del Estado de Morelos, de los cuales 18 (35.3\%) correspondían al sexo masculino y 33 (64.7\%) al sexo femenino, 19 personas cursaban el cuarto semestre y 32 el séptimo semestre, 4 de ellos se identifican en el nivel socioeconómico bajo, 34 en el nivel medio, y 13 en el nivel socioeconómico alto. De acuerdo con los datos obtenidos por medio del IAAR se concluye que 37 de los estudiantes presentan resiliencia en sus diferentes niveles y 14 de ellos presentan anomia, la cual se caracteriza como ausencia de resiliencia (Ver tabla 1).

\section{Tabla 1}

Características de los participantes

\begin{tabular}{lcc}
\hline Género & F & $\mathbf{\%}$ \\
\hline Masculino & 18 & 35.3 \\
Femenino & 33 & 64.7 \\
Semestre & & \\
Cuarto semestre & 19 & 37.3 \\
Séptimo semestre & 32 & 62.7
\end{tabular}

Nivel socioeconómico

Bajo

Medio

Alto

Niveles de anomia y resiliencia.

Anomia muy alta

Anomia Alta 


\begin{tabular}{lcc}
\hline Anomia media & 6 & 11.8 \\
Anomia baja & 6 & 11.8 \\
Resiliencia media & 10 & 19.6 \\
Resiliencia alta & 15 & 29.4 \\
Resiliencia muy alta & 12 & 23.5 \\
Rendimiento Académico & & \\
Medio & 19 & 37.3 \\
Alto & 32 & 62.7 \\
\hline
\end{tabular}

De acuerdo con los objetivos del estudio se muestran los resultados de la PCL obtenidos entre la variable resiliencia y género por medio de la prueba de Speerman, en la que se evidencia una correlación de $\mathrm{r}=-.319, \mathrm{y} \mathrm{p}=.023$. y en la relación mediante la PCL de Speerman, la variable resiliencia y el RA tuvieron una correlación estadísticamente significativa ( $r=0.344, \mathrm{p}=0.013$; ver tabla 2).

Tabla 2

Correlación entre la resiliencia, género y rendimiento académico

\begin{tabular}{lcc}
\hline $\mathbf{N = 5 1}$ & $\mathbf{r}$ & $\mathbf{p}$ \\
\hline Género & -0.319 & 0.023 \\
& & \\
Rendimiento & 0.344 & 0.013 \\
académico & &
\end{tabular}

Nota: $r=$ correlación; $p=$ nivel de significancia

*Prueba de Speerman

Una vez obtenida la PCL se procedió a realizar una prueba estadística no paramétrica de comparación intergrupal entre la resiliencia y el género por medio de la prueba U de Mann-Whitney para variables independientes, con lo cual se ultimó que los hombres presentan un promedio mayor $(M=30.00)$, a comparación de las mujeres $(\mathrm{M}=23.82)$ en los resultados de resiliencia, diferencias que fueron estadísticas significativas $\quad(\mathrm{U}=2.25$, $\mathrm{p}=0.024)$. Por otro lado, después de obtener la PCL de la resiliencia y el RA se prosiguió a una comparación intergrupal entre la resiliencia y el RA, en la cual se obtuvo que los estudiantes con RA más alto presentaban en sus resultados de resiliencia un promedio mayor $(M=29.92)$ en comparación con los estudiantes de RA medio ( $M=20.92)$, es importante mencionar que, en esta población ninguno de los participantes tenía un rendimiento académico bajo. Por último, en relación con estas variables se encontró una correlación 
estadísticamente significativa $(\mathrm{U}=207.5$, $\mathrm{p}=0.015$; ver tabla 3 ).

Tabla 3

Comparación de la resiliencia por género y rendimiento académico

\begin{tabular}{lcccc}
\hline Variable & N & Media & U & p \\
\hline Género & 18 & 30.00 & 225.00 & 0.024 \\
Hombre & & & & \\
Mujer & 33 & 23.82 & & \\
Rendimiento académico & 19 & 20.92 & 207.5 & 0.015 \\
Medio & & & & \\
Alto & 32 & 29.92 & & \\
\hline
\end{tabular}

Nota: $\mathrm{U}=\mathrm{U}$ de Mann-Whitney; N=número de personas; $\mathrm{p}=$ nivel de significancia

Así mismo se obtuvieron las correlaciones entre los ítems del IAAR y el RA, entre los cuales se encontraron, 11.- Me gusta mi forma de ser $(\mathrm{r}=-0.325, \mathrm{p}=0.020)$ en donde los estudiantes que presentan RA medio indican que les gusta su forma de ser, en el ítem 13.- Prefiero que me digan lo que debo de hacer ( $\mathrm{r}=-0.348, \mathrm{p}=0.012)$, se puede ver que los estudiantes que presentan mayor RA se identifican frecuentemente con esta afirmación, indicando que les gusta más que les digan qué debe de realizar, en el ítem 18.- Yo soy capaz de trabajar en equipo ( $\mathrm{r}=-0.279, \mathrm{p}=0.047$ ), los datos indican que los estudiantes con RA medio indican que son capaces de trabajar en equipo. En el ítem 19.- Aunque tengo algunos defectos me acepto como soy $(\mathrm{r}=-$ 0.299, $\mathrm{p}=0.033$ ), se evidencia que, entre mayor sea el RA, presentan más dificultades para aceptarse tal y como son, respecto al ítem 23.-Cuando hay problemas o dificultades me cuesta trabajo resolverlos ( $\mathrm{r}=0.500, \mathrm{p}=0.0000$ ), se puede notar que los estudiantes con mayor RA presentan mejor dominio frente a las problemáticas o dificultades que puedan llegar a surgir y por último, el ítem 32.- Me doy cuenta de que cuando tengo problemas soy capaz de aprender y ser más fuerte $(\mathrm{r}=-0.076, \mathrm{p}=0.29)$ los estudiantes con RA medio indicaron que son capaces de aprender de experiencias pasadas y poder salir adelante (Ver tabla 4 ). 
Tabla 4

Correlación de los ítems de resiliencia y el rendimiento académico

\begin{tabular}{llllll}
\hline IT & $\mathbf{r}$ & $\mathbf{p}$ & $\mathbf{I T}$ & $\mathbf{r}$ & $\mathbf{p}$ \\
\hline 01 & -0.184 & 0.195 & 18 & -0.279 & 0.047 \\
02 & -0.234 & 0.099 & 19 & -0.299 & 0.033 \\
03 & -0.177 & 0.214 & 20 & -0.072 & 0.613 \\
04 & 0.016 & 0.910 & 21 & 0.273 & 0.053 \\
05 & -0.089 & 0.535 & 22 & 0.084 & 0.556 \\
06 & 0.159 & 0.264 & 23 & 0.500 & 0.000 \\
07 & -0.239 & 0.091 & 24 & 0.144 & 0.313 \\
08 & 0.056 & 0.695 & 25 & -0.011 & 0.940 \\
09 & 0.125 & 0.382 & 26 & -0.014 & 0.925 \\
10 & -0.145 & 0.311 & 27 & -0.202 & 0.156 \\
11 & -.325 & 0.020 & 28 & -0.077 & 0.592 \\
12 & -0.136 & 0.340 & 29 & -0.156 & 0.274 \\
13 & -.348 & 0.012 & 30 & -0.034 & 0.811 \\
14 & 0.192 & 0.177 & 31 & -0.044 & 0.761 \\
15 & -0.039 & 0.785 & 32 & -0.076 & 0.296 \\
16 & -0.177 & 0.213 & 33 & -0.098 & 0.496 \\
17 & -0.144 & 0.314 & 34 & 0.079 & 0.581 \\
\hline
\end{tabular}

Por otra parte, también se correlacionaron los ítems del IAAR con la variable género, encontrando una correlación significativa en el ítem 6.- Me cuesta trabajo tomar mis propias decisiones ( $\mathrm{r}=-0.437, \mathrm{p}=0.001)$, 31.- Cuando una persona tiene algún defecto me burlo de ella $(\mathrm{r}=0.291, \mathrm{p}=0.039)$, por lo cual se puede concluir que, a los estudiantes mujeres les cuesta trabajo tomar sus propias decisiones. El género femenino rechaza mayormente el burlarse de los demás por algún defecto (Ver tabla 5). 
Tabla 5

Correlación de los ítems de resiliencia y el género

\begin{tabular}{llllll}
\hline IT & $\mathbf{r}$ & $\mathbf{p}$ & $\mathbf{I T}$ & $\mathbf{r}$ & $\mathbf{p}$ \\
\hline 01 & 0.214 & 0.132 & 18 & 0.092 & 0.523 \\
02 & 0.244 & 0.084 & 19 & 0.141 & 0.323 \\
03 & 0.158 & 0.267 & 20 & 0.184 & 0.196 \\
04 & -0.137 & 0.338 & 21 & 0.166 & 0.245 \\
05 & -0.012 & 0.935 & 22 & -0.078 & 0.587 \\
06 & -0.437 & 0.001 & 23 & -0.111 & 0.439 \\
07 & 0.208 & 0.143 & 24 & 0.032 & 0.822 \\
08 & 0.124 & 0.386 & 25 & 0.093 & 0.514 \\
09 & -0.010 & 0.946 & 26 & -0.014 & 0.924 \\
10 & 0.170 & 0.233 & 27 & 0.171 & 0.230 \\
11 & 0.067 & 0.642 & 28 & 0.071 & 0.619 \\
12 & 0.276 & 0.050 & 29 & 0.124 & 0.386 \\
13 & 0.114 & 0.427 & 30 & 0.053 & 0.711 \\
14 & -0.154 & 0.280 & 31 & 0.291 & 0.039 \\
15 & 0.177 & 0.213 & 32 & 0.199 & 0.162 \\
16 & -0.020 & 0.890 & 33 & -0.173 & 0.224 \\
17 & 0.020 & 0.888 & 34 & 0.276 & 0.050 \\
\hline & & & & &
\end{tabular}

\section{Discusión}

Según el resultado general los niveles más altos de resiliencia pertenecen al sexo masculino, donde se puede observar una correlación estadísticamente significativa, lo cual concuerda con los resultados encontrados por Ramírez \& Castro (2018) quienes evidencia que, el sexo masculino es el que presentó valores medios más altos en resiliencia que los del sexo femenino, tal y como sucede en esta investigación. Por el contrario, Grotberg
(1995) encontró que, en los niveles de resiliencia, el género es una variable que presenta diferencias significativas siendo las mujeres más protectoras; y según González-Arratia et al., 2008; Morales \& Díaz, 2011; Valdez, 2013) en su mayoría se ve asociada con la resiliencia, incluso en los diferentes grupos de edad.

Estos resultados se debería a que al parecer las mujeres, son también, las que requieren de un apoyo externo, principalmente en la familia para ser resilientes, suceso que en este estudio es difícil de observar debido a que la mayor parte de las mujeres que cursan la carrera 
de nutrición proviene de diferentes municipios, incluso de diferentes Estados de la república mexicana, por lo cual, el traslado resulta difícil y sus opciones se reducen a obtener algún departamento en renta cerca de la institución universitaria, pero lejos de sus familias y sus lugares de origen.

Por su parte, Villalta \& Saavedra (2012) reportan también, que las mujeres presentan un promedio más elevado en resiliencia que los hombres, aunque no se presentaron significancias en sus datos.

En esta investigación son los hombres los cuales presentaban mayor dominio sobre la capacidad resiliente, tal caso podría deberse a que los estudiantes hombres tienden a ser más independientes al verse lejos de su familia, socializando de una manera efectiva, al contrario de las mujeres, que al estar lejos del seno familiar se sienten más vulnerables, ya que, por cuestiones de la cosmovisión mexicana las mujeres suelen permanecer dentro de la familia.

Al igual que en este estudio, las indagaciones realizadas por Ramírez y Castro (2018), Finez y Morán (2014) muestran que el sexo femenino tiende a tener menores niveles de resiliencia a comparación con los varones, los cuales presentaron más factores protectores, además de tener mayor dominio al enfrentarse a los contratiempos, fue así como estos autores concluyeron que, aunque actualmente nos encontramos en una sociedad cada vez más igualitaria, existen diferencias en cada género.
La resiliencia es un tema que se debe de estudiar cuidadosamente en cada una de las áreas de investigación, especialmente es importante que se ponga cuidado meticuloso en la relación que existe entre la resiliencia y el género, ya que la población llega a presentar niveles de resiliencia diferentes debido a su cosmovisión, costumbres, etc., tal y como lo sostiene Cho (2014) quien afirma que, existe una relación entre las dimensiones de resiliencia y el contexto.

En cuanto a los datos obtenidos por medio de la PCL entre la resiliencia y el RA se encontró una correlación estadísticamente significativa al igual que los estudios de Álvarez (2010), Atencia (2020), Huaire (2014), León et al. (2019), Pizarro (2017) y Villalta (2009), donde los estudiantes presentaron correlaciones entre el nivel de resiliencia y RA (calificaciones acumuladas), esto también sr presentan en niños de 5to y 6to grado de primaria (Gallesi, 2012).

En otro estudio realizado por Peralta, Ramírez \& Castaño (2006) se evidencia que existe una correlación estadísticamente significativa entre la resiliencia $y$ el RA de estudiantes universitarios, siendo los estudiantes de menor RA quienes se encontraban con niveles más bajos de resiliencia, esto podría deberse a lo que varios investigadores han formulado a través de los años acerca de la resiliencia académica, donde los estudiantes con mayores niveles de resiliencia son capaces de tener RA más alto, pues presentan metas encaminadas a su futuro. Por su parte, Gómez (2013) encontró que eran los estudiantes con 
mayor resiliencia positiva los cuales poseían las calificaciones más altas, agrego de igual modo que los estudiantes con menor RA poseían resiliencia negativa, al contrario de las investigaciones previamente planteadas.

Gaxiola, Ramírez y Contreras (2012), también evidenciaron que los jóvenes adolescentes de un bachillerato situado en una zona de alta vulnerabilidad, los que presentan mayor RA también poseían altos índices de resiliencia además que, estas dos variables se asociaban a tener metas claras encaminadas a su futuro.

\section{Conclusiones}

Por conclusión, se agrega que los resultados obtenidos en esta investigación son un aporte significativo para el estudio de la resiliencia, en relación con el RA, y el género. En tanto, la correlación existente entre resiliencia y género se concluye que los hombres obtuvieron mayores índices de resiliencia por lo cual es importante que se promuevan acciones que vayan encaminadas a poder intervenir y aumentar la resiliencia de los estudiantes que viven lejos de su lugar de origen y de sus familias. Existen investigaciones previamente realizadas que han tenido efectos de entrenamiento positivos en resiliencia $y$ que bien podrían ayudar a promover esta capacidad en el sexo femenino, ya que algunos investigadores han sostenido que el contexto si impacta en los niveles de resiliencia de ambos sexos, dependiendo de las circunstancias.

Por otra parte, es importante destacar que se necesitan más investigaciones que intenten relacionar la resiliencia con el RA (Calificaciones acumuladas) en población universitaria, ya que la mayor parte de investigaciones se ven desarrolladas en estudiantes que cursan los niveles básicos. Sin embargo, si podemos agregar que la resiliencia resulta una capacidad que se debe desarrollar dentro de los espacios educativos, ya que presentan relaciones positivas con el RA como lo demostró esta y otras indagaciones y tesis en relación con este tema de investigación.

\section{Referencias}

Álvarez, R., \& Cáceres, L. (2010). Resiliencia, rendimiento académico y variables sociodemográficas en estudiantes universitarios de Bucaramanga (Colombia). Psicología iberoamericana, 18(2), 37-46. https://www.redalyc.org/pdf/133 9/133915921005.pdf
Atencia, M., \&Hernández, E. (2020). Resiliencia, burnout y fracaso académico en estudiantes de Ingeniería de la Universidad de Córdoba, Colombia. Revista Espacios, 41(11), 23-39. https://www.revistaespacios.com/ a20v41n11/20411123.html 
Castillo, A., et al. (2017). Edad, género y resiliencia en la conducta sexual en riesgo para ITS en adolescentes al sur de México. Revista electrónica trimestral de enfermería, 45(1), 168177.

http://scielo.isciii.es/pdf/eg/v16n 45/1695-6141-eg-16-4500168.pdf

Cho, M. (2014). The influence of family's participation in recreational sports on its resilience and communication facilitation. Journal Exercise rehabilitation, 10 (5), 313-318. https://doi.org/10.12965/jer.1401 $\underline{61}$

Coronado, H. A. (2017). Alumnado académicamente resiliente. En A. Jiménez-Hernández (EDS.). Reconstruyendo un mundo con ojos de niñas (pp. 2806-2815). GEU.

Ferrer, J. (2011). Autoconcepto académico y resiliencia en universitarios de alto rendimiento: construcción desde una perspectiva biográfica. Anuario del Doctorado en Educación: Pensar la Educación, (5), 40-63. http://erevistas.saber.ula.ve/index. php/anuariodoctoradoeducacion/a rticle/\%20view/3812/3\%20649

Fínez, M. J. \& Morán, C. (2014). Resiliencia y autoconcepto: su relación con el cansancio emocional en adolescentes. Revista INFAD de Psicología. International Journal of Developmental and Educational Psychology, 6(1), 289-296. https://doi.org/10.17060/ijodaep. 2014.n1.v6.746.
Flores, D. (2013). La resiliencia nómica: Una nueva realidad para personas que logran lo que se proponen. Autor, Editor.

Gallesi, R. G. (2012). Resiliencia y rendimiento académico en estudiantes de 5to y 6to grado de primaria. Revista IIPSI, 15(1), 181201.

https://doi.org/10.15381/rinvp.v1 $\underline{5 i 1.3677}$

Gaxiola, R, J. C., González-L, S., \& Contreras, H, Z. G. (2012). Influencia de la resiliencia, metas y contexto social en el rendimiento académico de bachilleres. Revista electrónica de investigación educativa, 14(1), 165181.

Gaxiola, R. J., Gonzales-Lugo, S., Contreras, H. Z., \& Gaxiola V. E. (2012). Predictores del rendimiento académico en adolescentes con disposiciones resilientes y no resilientes. Revista de Psicología, 30(1), 51-58. http://www.scielo.org.pe/scielo.ph p?script $=$ sci arttext\&pid $=$ S025492472012000100003\&lng=es\&tlng =es.

Grotberg, E. (1995). Una guía para promover la resiliencia en niños: fortaleciendo el espíritu humano, proyecto internacional de la resiliencia. La Haya: Bernard Van Leer Fundation.

Gómez, D., Moreno, J., Cruz, L., \& Ortiz, M. (2019). El concepto de la resiliencia a través de la historia. Revista de 
sociología contemporánea, 6(19), 16-19.

Revista de Sociología Contemporá nea V6 N19 3.pdf (ecorfan.org)

Gómez, E. (2013). La resiliencia y su correlación con la depresión y el RA en estudiantes de la licenciatura en pedagogía de la universidad privada del Estado de México, Campus TEMAC, septiembre 2011 - Julio 2012 [Tesis de posgrado, Universidad Autónoma del Estado de México]. $\quad$ 394684.pdf (uaemex.mx)

Gómez, G., \& Rivas, M. (2017). Resiliencia académica nuevas perspectivas de interpretación del aprendizaje en contextos de vulerabilidad social. Calidad en la educación, 6(47), 215233.

https://doi.org/10.31619/caledu.n $\underline{47.35}$

González-Arratia, N. (2009). Resiliencia y salud en niños y adolescentes. Ciencia ergo-sum, 16(3), 247-253.

Gónzales- Arriata, N \& Valdez, J. (2013). Resiliencia y diferencias por edad en hombres y mujeres mexicanas. Acta de investigación psicológica, 3 (1), 941- 955. https://doi.org/10.1016/S20074719(13)70944-X

Huaire, E. (2014) Prevalencia de resiliencia y autoestima sobre el rendimiento escolar en estudiantes de instituciones educativas de Ate Vitarte, Lima. Apuntes de ciencia y sociedad, 4 (2), 202-209. https://doi.org/10.18259/acs.201 $\underline{4023}$

Jhonson, D., \& Jhonson, R. (2002). Teaching Students How to Cope with Adversity: the Three Cs en Frydenberg. Beyond Coping. Meeting goals, 1(1), 195-216 https://psycnet.apa.org/record/20 $\underline{03-06485-010}$

León, A., González-Escobar, S., González., López, N. I., \& Barcelata, B. (2019). Estrés autoeficacia, rendimiento académico y resiliencia en adultos emergentes. Electronic Journal of Research in Educational Psychology http://hdl.handle.net/20.500.1179 $\underline{9 / 105081}$

Llobet, V. (2008). La promoción de la resiliencia con niños y adolescentes, entre la vulnerabilidad y la exclusión, Herramientas para la transformación. NOVEDUC. https://books.google.com.mx/book s?id=ZCCphed Ja4C\&pg=PA87\&lpg =PA87\&dq=La+resiliencia+no+es+ un+rasgo+de+personalidad, + sino+ que+las+personas+son+actores+y+ fuentes+de+las+adaptaciones+resil ientes\%E2\%80\%9D\&source=bl\&ot $\mathrm{s}=\mathrm{aQCm} 0 \mathrm{pBzLq} \& \mathrm{sig}=\mathrm{ACfU} 3 \mathrm{U} 2-$ xtj2IlYHL1FjPKgQ

López, S. A., \& Ubals, A. J. (2012). La resiliencia un acercamiento viable desde la práctica educativa. EduSol, 12(41), 43-51. La resiliencia: un acercamiento viable desde la práctica educativa - Dialnet (unirioja.es) 
Lyn, W. (2014). Building resilience in three Australian high school using resilience doughnut framework. En, S. Prince, \& D, Saklofske, Resilience Interventions for Youth in Diverse Populations (pp. 217-257). https://doi.org/10.1007/978-14939-0542-311.

Martin, A. J. \& Marsh, H. W. (2008). Academic buoyancy: Towards an understanding of students' everyday academic resilience. Journal of school psychology, 46(1), 53-83.

https://www.sciencedirect.com/sc ience/article/pii/S0022440507000 131\#!

Morales, M. \& Díaz, D. (2011). Estudio comparativo de la resiliencia en adolescentes: el papel del género, la escolaridad y procedencia. Uaricha, 8(17), 62-77.

Ovejero, M. (2018). Evaluación de fortalezas humanas en estudiantes de la Universidad Complutense de Madrid y diferencias de sexo: Relación con salud, resiliencia y rendimiento académico [Tesis doctoral, Universidad Complutense de Madrid].

Peralta, S., Ramirez, A., y Castaño, H. (2006). Factores resilientes asociados al rendimiento académico en estudiantes pertenecientes a la universidad de Sucre Barranquilla (Colombia), Psicología desde el caribe, 17 (1) 196-219.
Pinel, M., \& Carrión, M. (2019). Relación entre género, resiliencia $\mathrm{y}$ autoconcepto académico y social en la adolescencia. Revista de Psicología y Educación, 14(2), 112123.

http://www.revistadepsicologiaye ducacion.es/pdf/176.pdf

Pintado, S., \& Cruz, F. (2017). Factores de resiliencia en niños y adolescentes con cáncer y su relación con el género y la edad. Ciencia Ergo-Sum, $242,137-144$.

Pizarro, C. (2017). Relación entre autoestima, resiliencia y rendimiento académico en estudiantes del Centro Preuniversitario de la Universidad Nacional Mayor de San Marcos (CEPREUNMSM).

https://hdl.handle.net/20.500.126 $\underline{72 / 6624}$

Prado, A., \& Águila, C. (2013). Diferencia en la resiliencia según género y el nivel socioeconómico en adolescentes. Persona, 6, 179-196. https://www.redalyc.org/articulo. oa?id=147118110009

Ramírez, I., \& Castro, M. (2018). Análisis de los niveles de resiliencia en función del género y factores del ámbito educativo en escolares. Eshpa. 2 (1), 50-60.

Salgado, L. A. (2005). Métodos e instrumentos para medir la resiliencia: Una alternativa peruana. Liberabit, 11(11), 41-48. http://pepsic.bvsalud.org/pdf/libe rabit/v11n11/v11n11a06.pdf 
Scafarelli, T. L., \& García-Pérez, R. (2010). Estrategias de afrontamiento al estrés en una muestra de jóvenes universitarios uruguayos. Ciencias psicológicas, $\quad 4(2), \quad$ 165-175. http://www.scielo.edu.uy/scielo.p

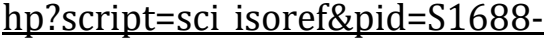
42212010000200004\&lng=es\&tlng =es

Villalta, P. M. (2010). Factores de resiliencia asociados al rendimiento académico en estudiantes de contextos de alta vulnerabilidad. Revista de pedagogía, 31 (88), 159-
188.

https://www.redalyc.org/articulo. oa?id=65916617007

Worsley, L. (2015). The resilience doughnut: combining strengths to survive. En M. Claire Patron, \& S. Holden, Victim victorious: from fire to phoenix. (pp. 67-87). Nova Science

Publishers https://researchers.mq.edu.au/en/ publications/the-resiliencedoughnut-combining-strengths-tosurvive. 
Apéndice

Test de resiliencia y asiliencia nomica

\begin{tabular}{|c|c|c|c|c|}
\hline & Nombre: & $\mathrm{Si}$ & $\begin{array}{c}\text { A } \\
\text { veces }\end{array}$ & No \\
\hline 1 & Estoy de buen humor, aunque tenga problemas. & & & \\
\hline 2 & Puedo tomar decisiones con facilidad. & & & \\
\hline 3 & Tengo confianza en mí mismo. & & & \\
\hline 4 & Me esfuerzo por decir la verdad y que me entiendan. & & & \\
\hline 5 & Me gusta buscar caminos nuevos para llegar a una meta. & & & \\
\hline 6 & Me cuesta trabajo tomar mis propias decisiones. & & & \\
\hline 7 & Puedo resolver lo difícil. & & & \\
\hline 8 & $\begin{array}{l}\text { Ante un problema difícil trato de estar tranquilo y busco resolver la } \\
\text { situación. }\end{array}$ & & & \\
\hline 9 & Para lograr lo que quiero trato de no hacer trampa. & & & \\
\hline 10 & Me recupero bien después de estar triste. & & & \\
\hline 11 & Me gustan mi forma de ser. & & & \\
\hline 12 & Puedo buscar maneras de resolver mis problemas. & & & \\
\hline 13 & Prefiero que me digan lo que debo hacer. & & & \\
\hline 14 & Me disgusta mi cuerpo y lo rechazo. & & & \\
\hline 15 & Puedo resolver problemas propios de mi edad. & & & \\
\hline 16 & Me siento bien con los compañeros de mi clase. & & & \\
\hline 17 & Aunque a veces deseo hacer algo prohibido, puedo evitarlo. & & & \\
\hline 18 & Yo soy capaz de trabajar en equipo. & & & \\
\hline 19 & Aunque tengo algunos defectos me acepto como soy. & & & \\
\hline 20 & Es difícil que me dé por vencido cuando hay que terminar algo. & & & \\
\hline 21 & $\begin{array}{l}\text { Cuando me piden que entregue mi tarea generalmente no la termino } \\
\text { a tiempo. }\end{array}$ & & & \\
\hline 22 & Si hay algo que hacer, no me tienen que decir que lo haga. & & & \\
\hline
\end{tabular}




\begin{tabular}{|l|l|l|l|}
\hline 23 & Cuando hay problemas o dificultades, me cuesta trabajo resolverlos. & & \\
\hline 24 & Cuando hay peligro no se prevenirlo. & & \\
\hline 25 & Me enojo sin lastimar a nadie. & & \\
\hline 26 & Me gusta que los demás tomen las decisiones por mí. & & \\
\hline 27 & Manejo bien mis sentimientos cuando no obtengo lo que quiero. & & \\
\hline 28 & $\begin{array}{l}\text { Logro acostumbrarme a las situaciones que cambian y no me } \\
\text { detengo ante los problemas. }\end{array}$ & & \\
\hline 29 & Me gusta ayudar cuando alguien está triste o tiene problemas. & & \\
\hline 30 & Ayudo a mis compañeros cuando puedo. & & \\
\hline 31 & Cuando una persona tiene algún defecto me burlo de ella. & & \\
\hline 32 & Me doy cuenta de que cuando tengo problemas soy capaz de & & \\
\hline 33 & Me veo incapaz de lograr algo importante. & & \\
\hline
\end{tabular}

